International Journal of Modern Physics B (C) World Scientific Publishing Company

\title{
The dynamo bifurcation in rotating spherical shells
}

\author{
VINCENT MORIN \& EMMANUEL DORMY \\ MAG (CNRS/ENS/IPGP), Ecole Normale Supérieure, \\ 24 rue Lhomond, 75252 Paris Cedex 05, France* \\ vincent.morin@ens-lyon.fr,dormy@phys.ens.fr
}

Received 21 Oct. 2009

\begin{abstract}
We investigate the nature of the dynamo bifurcation in a configuration applicable to the Earth's liquid outer core, i.e. in a rotating spherical shell with thermally driven motions. We show that the nature of the bifurcation, which can be either supercritical or subcritical or even take the form of isola (or detached lobes) strongly depends on the parameters. This dependence is described in a range of parameters numerically accessible (which unfortunately remains remote from geophysical application), and we show how the magnetic Prandtl number and the Ekman number control these transitions.
\end{abstract}

Keywords: Dynamo theory; Bifurcations; Instabilities.

\section{Introduction}

The origin of the Earth magnetic field remains a challenging issue for physicists. The accepted theory, known as dynamo theory, describes the transfer from kinetic to magnetic energy as an instability process. Above a given threshold electrical currents, and thus magnetic fields, are amplified by a turbulent flow of a conducting fluid.

This problem can be described in its simpler form by a set of coupled partial differential equations (see section 2). The state of this system is fully characterised by four independent controlling parameters. The Ekman number E, which can be interpreted as measuring the ratio of the length of the day to a typical viscous timescale, this number is extremely small in the case of the Earth's core (the Earth is in rapid rotation). The magnetic Prandtl number Pm, measuring the ratio of a typical ohmic timescale to viscous timescale, it is a characteristic of the fluid and is minute for all liquid metals (including liquid iron in the Earth's core). The hydrodynamic Prandtl number Pr, also characterizing the fluid and which compares a thermal timescale to a viscous timescale, this number is of order one, or slightly less, but non-vanishing. Finally the Rayleigh number Ra, which measures a ratio

*VM is now at: Laboratoire de Physique, Ecole Normale Supérieure de Lyon, CNRS UMR5672, 46 allée d'Italie, F-69364 Lyon, France. 
of driving forced to forced slowing down the motion. Its value is difficult to assess in a simple Boussinesq model. This will be the most obvious controlling parameter, which needs to be varied to investigate dynamo properties.

Kutzner and Christensen [1] performed a first systematic exploration of the parameter space available to numerical models of the geodynamo. They produced a "phase diagram" for dynamo action in terms of Ekman number E and magnetic Prandtl number (or equivalently here Roberts number, $\mathrm{q}=\mathrm{Pm} / \mathrm{Pr}$ ) and Rayleigh number Ra. Their study was later extended to lower values of $\mathrm{E}$ and $\mathrm{Pm}$ in [2]. We present a schematic sketch of their results in figure 1 (See also the $\mathrm{PhD}$ thesis of Carsten Kutzner [3]). Each diagram corresponds to a given Ekman number, ranging here from $2 \cdot 10^{-2}$ to $10^{-4}$. Kutzner and Christensen identified three "phases" on these diagrams. In part of the parameter space, no dynamo solutions were obtained. In the complementary region, in which dynamo is obtained, two regimes are identified. One at low forcing, characterised by strongly dipolar, but non-reversing dynamos, and another corresponding to reversing, but multipolar dynamos. This, of course, is not a very good news when comparing these models with the geodynamo (characterized by a reversing strongly dipolar field).

As pointed above, all models presented in these diagrams suffer from parameters extremely remote from their geophysically relevant estimates. In particular, the Ekman number $\mathrm{E}$ is over-estimated by a factor in excess of a billion and the magnetic Prandtl number Pm by a factor close to a million. In fact this last number offers a ratio of diffusivity which is equal to unity or larger in the numerics, whereas it should be as small as $10^{-6}$ in the Earth. The diagram reveals that if the magnetic Prandtl number is decreased in numerical models (all other parameters being fixed), dynamo action is lost! This does not come as a surprise, as it corresponds to increasing the ohmic resistivity, but appears to prevent any direct comparison of the models with the actual geodynamo.

In fact the main result derived from these phase diagrams is much more optimistic. The authors noted that the threshold value $\mathrm{Pm}_{L}$, below which dipolar dynamos are lost decreases with the Ekman number. They proposed [4, 2] a scaling in the form $\operatorname{Pm}_{L} \propto \mathrm{E}^{\alpha}$ and proposed a numerical fit to $\alpha \simeq 3 / 4$ (see [5] for a theoretical explanation of this behaviour leading to $\alpha \simeq 2 / 3$ ). Which could indicate a connection in the parameter space between the models presently available and the regime relevant to the geodynamo.

Part of the Kutzner \& Christensen results have also been discussed in [4]. In this earlier study, the authors proposed that for $\mathrm{E}=10^{-3}$ the $\mathbf{B}=\mathbf{0}$ state is always stable. The corresponding bifurcations are therefore interpreted as subcritical by the authors. For $\mathrm{E}=10^{-4}$, the authors identify only supercritical bifurcations (because they find $\mathbf{B}=\mathbf{0}$ to be unstable). They however did not perform a systematic study to characterise the dynamo bifurcation, nor determined how it is controlled by the governing parameters. This is the object of the work reported here.

We present here a review of the results obtained and first described in french in Morin 2005 [6] (available online). These results were subsequently presented at 


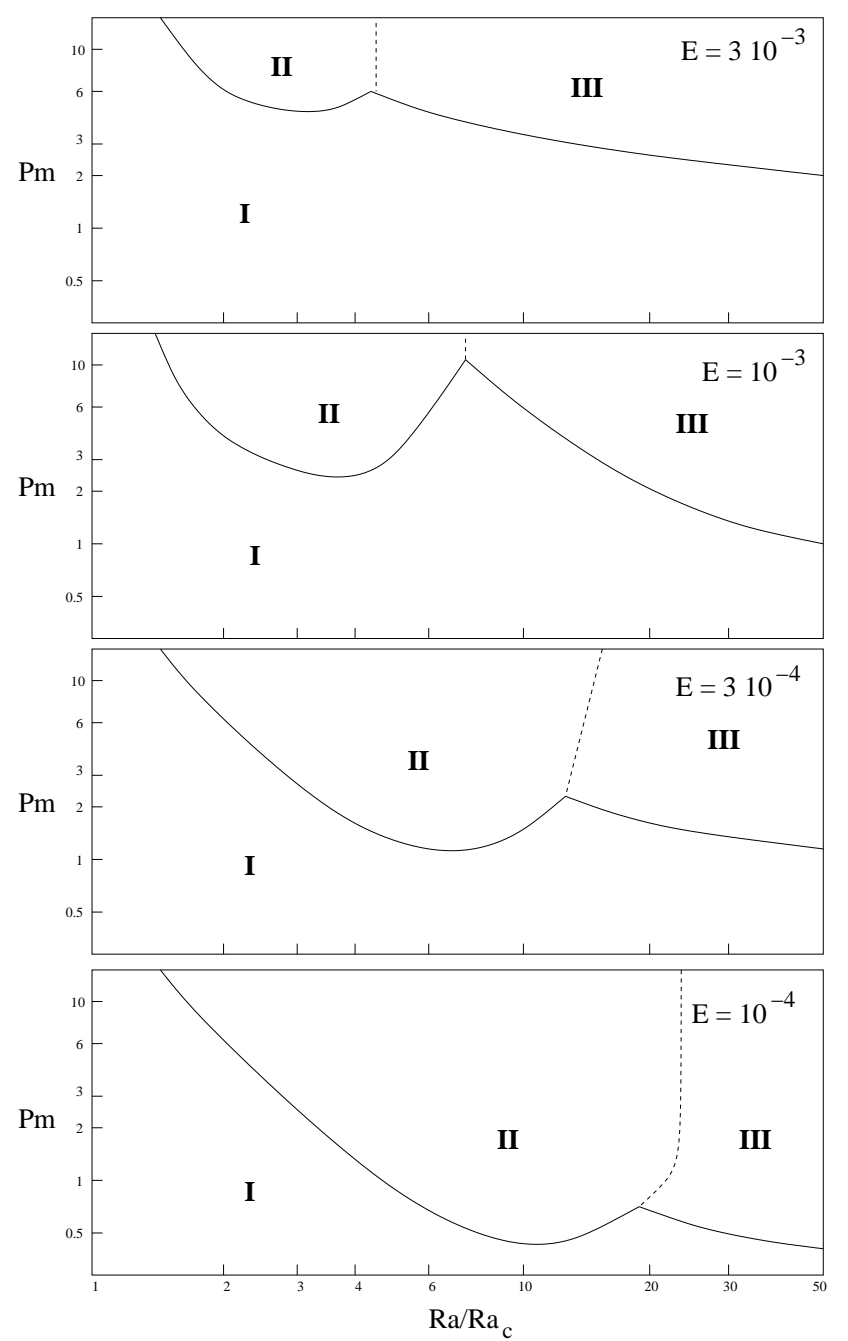

Fig. 1. "Phase diagram" for dynamo action for decreasing values of the Ekman number (after Kutzner, 2003). Three domains are identified. Domain I corresponds to parameters for which no dynamo solutions were obtained. Domain II corresponds to dynamo solutions dominated by a strong axial dipole. Domain III corresponds to reversing multipolar dynamos.

several international conferences 7 . They were not published in english so far, but have probably inspired more recent articles 8 . We should finally stress that while our results were originally obtained using only the numerical code call "Magic" (developed by G. Glatzmaier, U. Christensen and J. Wicht), we have since then reproduced these results using the local "PaRoDy" code. Simulations with this second code have confirmed the results presented here. 


\section{Numerical investigations}

In all the simulations presented here, we solve the MHD equations in the Boussinesq approximation in a rotating frame between two concentric spheres $\left(r_{o} / r_{i}=0.35\right)$. The reference frame is set such that the velocity vanishes on both spheres (no-slip boundaries), a temperature difference is maintained across the shell, and both the inner and the outer domain are assumed to be electrically insulating. The equations governing the solenoidal velocity $\mathbf{u}$ and magnetic $\mathbf{B}$ fields and the temperature $T$ can be written in non-dimensional form

$$
\begin{aligned}
& \mathrm{E}\left[\partial_{t} \mathbf{u}+(\mathbf{u} \cdot \boldsymbol{\nabla}) \mathbf{u}\right]=-\boldsymbol{\nabla} \pi+\mathrm{E} \Delta \mathbf{u}-2 \mathbf{e}_{z} \times \mathbf{u} \\
&+ \operatorname{Ra} T \mathbf{r}+\operatorname{Pm}^{-1}(\boldsymbol{\nabla} \times \mathbf{B}) \times \mathbf{B}, \\
& \partial_{t} \mathbf{B}=\boldsymbol{\nabla} \times(\mathbf{u} \times \mathbf{B})+\operatorname{Pm}^{-1} \Delta \mathbf{B}, \\
& \partial_{t} T+(\mathbf{u} \cdot \boldsymbol{\nabla}) T=\operatorname{Pr}^{-1} \Delta T,
\end{aligned}
$$

using the previously introduced Ekman number $\mathrm{E}=\nu / \Omega L^{2}$, the (modified) Rayleigh number $\mathrm{Ra}=\alpha g \Delta T L / \nu \Omega$, the Prandtl number $\operatorname{Pr}=\nu / \kappa$, and the magnetic Prandtl number $\mathrm{Pm}=\nu / \eta$, with $L=r_{o}-r_{i}$, and $g=g_{o} / r_{o}$ where $g_{o}$ is the gravity at $r=r_{o}$. For simplicity, we use here the same non-dimensional form as in [9. The rapid rotation of the reference frame is measured by the smallness of $\mathrm{E}$. In the following, the Prandtl number is set to unity, and thus $\mathrm{q} \equiv \kappa / \eta=\mathrm{Pm}$.

As we will present our results using $\mathrm{Ra} / \mathrm{Ra}_{c}$ as controlling parameter, where $\mathrm{Ra}_{c}$ is the critical Rayleigh number for the onset of thermal convection, we want to recall, for completeness, that the values of $\mathrm{Ra}_{c}$ are respectively 55.9, 60.8, and 69.7 for the Ekman numbers considered here i.e. $10^{-3}, 3 \cdot 10^{-4}$, and $10^{-4}$.

\subsection{Influence of the magnetic Prandtl number on the dynamo bifurcation}

Let us first fix the Ekman number and investigate the nature of the dynamo bifurcation varying the magnetic Prandtl number. With an Ekman number of $\mathrm{E}=3.10^{-4}$, when the magnetic Prandtl number is set to $\mathrm{Pm}=6$, the bifurcation diagram obtained numerically is reported on figure 2. We use filled dots to indicate stable solutions, and opened dots to indicate unstable solutions. The diagram reports magnetic energies, for varying values of the normalised Rayleigh number, dots indicate the time averaged values of the magnetic energy, while the standard deviation is indicated using error bars. The simulations are initialised with a small temperature perturbation, a vanishing velocity, and an infinitesimal magnetic perturbation.

One can note on figure 2 that the first point with non-zero magnetic field is obtained for $\mathrm{Ra}=2.57 \times \mathrm{Ra}_{c}$, the previous point on the diagram $\mathrm{Ra}=2.42 \times \mathrm{Ra}_{c}$, corresponds to a stable $\mathbf{B}=\mathbf{0}$ state. Figure 2 a demonstrates how the time averaged magnetic energy continuously tends to zero as he controlling parameter is decreased. This demonstrates the supercritical nature of this bifurcation. 


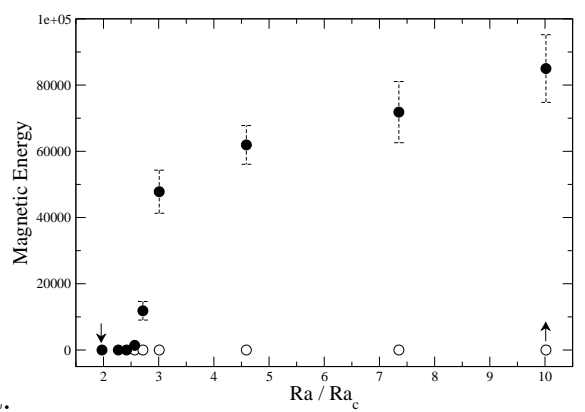

b.

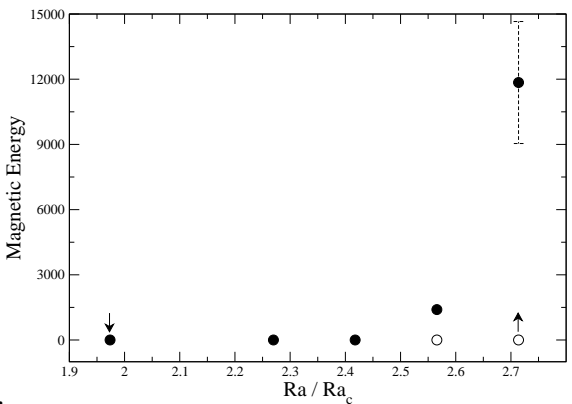

Fig. 2. Mean magnetic energy as a function of the Rayleigh number. The bifurcation is found to be supercritical for $\mathrm{E}=3 \cdot 10^{-4}$ and $\mathrm{Pm}=6$. Error bars indicate the standard deviation of energy fluctuations around the mean value. Graph b is an enlarged version of graph a, centered on the dynamo bifurcation.

The onset of dynamo action is here obtained close to the onset of convection. At the onset, the dynamo reaches a regime with constant kinetic and magnetic energy.

We should stress that each point on such diagram, corresponds to simulations of a few 100 of hours on a parallel computer. Computations are three-dimensional and need to be carried over a long enough time to obtain well converged averaged values.
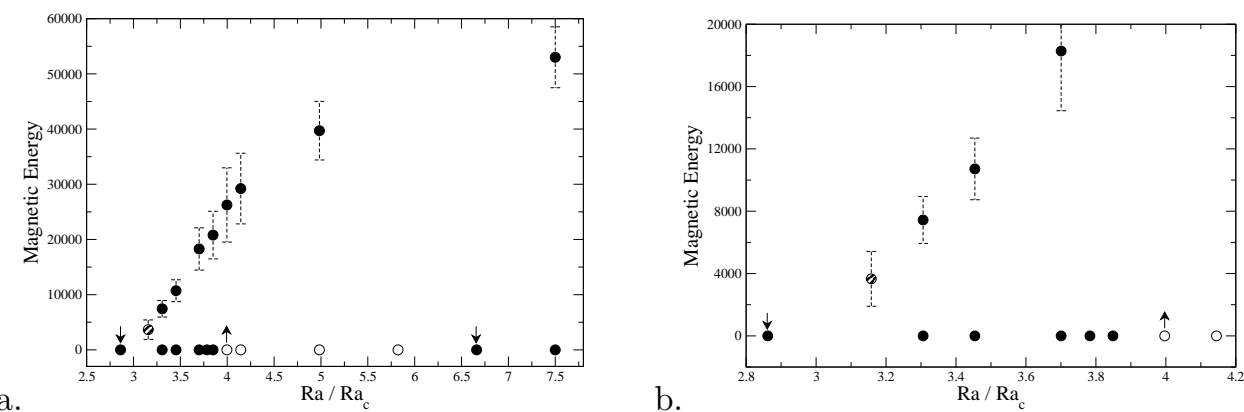

Fig. 3. Mean magnetic energy as a function of the Rayleigh number. This subcritical bifurcation was obtained for $\mathrm{E}=3 \cdot 10^{-4}$ and $\mathrm{Pm}=3$. Error bars indicate the standard deviation of the magnetic energy fluctuations around its mean value (dots). A close-up on the dynamo threshold is presented in graph $b$.

When the magnetic Prandtl number is decreased to $\mathrm{Pm}=3$, the onset of dynamo action occurs between $\mathrm{Ra}=3.85 \times \mathrm{Ra}_{c}$ and $\mathrm{Ra}=4 \times \mathrm{Ra}_{c}$ (further remote from the onset of convection). The magnetic energy reported on figure 3 exhibits an hysteretic behaviour characteristic of a subcritical bifurcation. The lower point on the subcritical branch is "metastable". It corresponds to $\mathrm{Ra}=3.16 \times \mathrm{Ra}_{c}$ and is designated on figure 3 by a stripped dot. By metastable, we mean a self-sustained dynamo solution, which could be maintained for a long time compared to viscous 
and ohmic diffusion times, but which eventually collapses when the system experiences a sufficiently large fluctuation (in the sense of a deviation from the mean magnetic energy). Once the dynamo solution is lost, the noise being multiplicative, the system remains in this state. In the case of this example, the dynamo solution was maintained for 40 viscous times, i.e. approximately 17 magnetic decay times before collapsing.

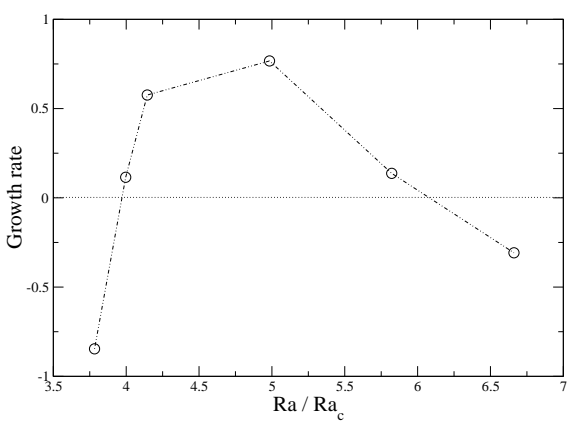

Fig. 4. Growthrate of the magnetic energy of a perturbation versus normalised Rayleigh number for $\mathrm{E}=3 \cdot 10^{-4}$ and $\mathrm{Pm}=3$. The growthrate of the solution first increases as the value of the Rayleigh number is increased above the dynamo threshold. It reaches a maximum value, and then decreases to eventually become negative. The hydrodynamic solution restabilises above $\mathrm{Ra} \simeq 6 \times \mathrm{Ra}_{c}$.

An intriguing characteristic of this bifurcation diagram is that the state $\mathbf{B}=\mathbf{0}$ restabilises for large enough values of the Rayleigh number, above the dynamo threshold. This is not due to an isolated point which behaves unexpectedly. In fact one can compute the linear growthrate of the magnetic field before reaching saturation, and the decay rate when $\mathbf{B}=\mathbf{0}$ is stable (see figure 4). This growthrate first increases above the onset, but then reaches a maximum as the Rayleigh number increases and eventually becomes negative again.

A third type of bifurcation is obtained for a magnetic Prandtl number of $\mathrm{Pm}=$ 1.5 (see figure 5 a). This bifurcation has an atypical shape sometimes referred to as "isola". In this regime, the $\mathbf{B}=\mathbf{0}$ solution does not destabilise, but a detached branch of dynamo solution exists for $\mathrm{Ra} / \mathrm{Ra}_{c} \in[4.44,9.18]$

We have observed in this section, decreasing the magnetic Prandtl number from $\mathrm{Pm}=6$ to $\mathrm{Pm}=1.5$, that we could describe supercritical, subcritical and isola diagrams for dynamo action. We shall note that the Rayleigh number had to be increased in this process (as the effects of Ohmic diffusion increase for decreasing values of the magnetic Prandtl number).

\subsection{Influence of the Ekman number on the dynamo bifurcation}

Rather than studying the parameter space at fixed Ekman number and varying the magnetic Prandtl number, we will now vary the Ekman number for a fixed magnetic 

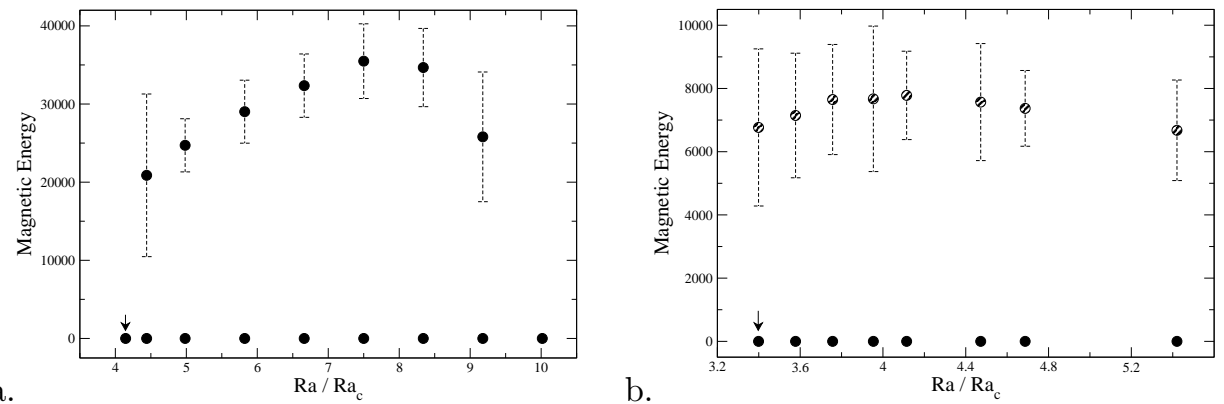

Fig. 5. Mean magnetic energy versus Rayleigh numbers. Isola diagram were obtained for $\mathrm{E}=$ $3.10^{-4}$ and $\mathrm{Pm}=1.5$ (left plot) and $\mathrm{E}=10^{-3}$ and $\mathrm{Pm}=3$ (right plot). Black dots (resp. stripped dots) indicate stable (resp. metastable) states. Error bars indicate the standard deviation of the magnetic energy fluctuations around its mean value. Metastable states are unstable to sufficiently large fluctuations of the velocity field. The probability for these fluctuations to occur are non zero. Purely hydrodynamic solutions were found to be always stable for this regime of parameters.

Prandtl number Pm $=3$. Results obtained for $\mathrm{E}=10^{-3}$ are reported on figure 5, b. This diagram corresponds again to an isola. All dynamo solutions were found to be metastable. The extreme points on this branch were maintained for roughly $6 \tau_{\nu}$ other points were maintained in time for up to $140 \tau_{\nu}$. We have seen previously that the dynamo bifurcation is subcritical for an Ekman number $\mathrm{E}=3 \cdot 10^{-4}$ and a magnetic Prandtl number $\mathrm{Pm}=3$. Let us now turn to $\mathrm{E}=10^{-4}$, the resulting bifurcation diagram is presented on figure 6. a and corresponds to a supercritical bifurcation.
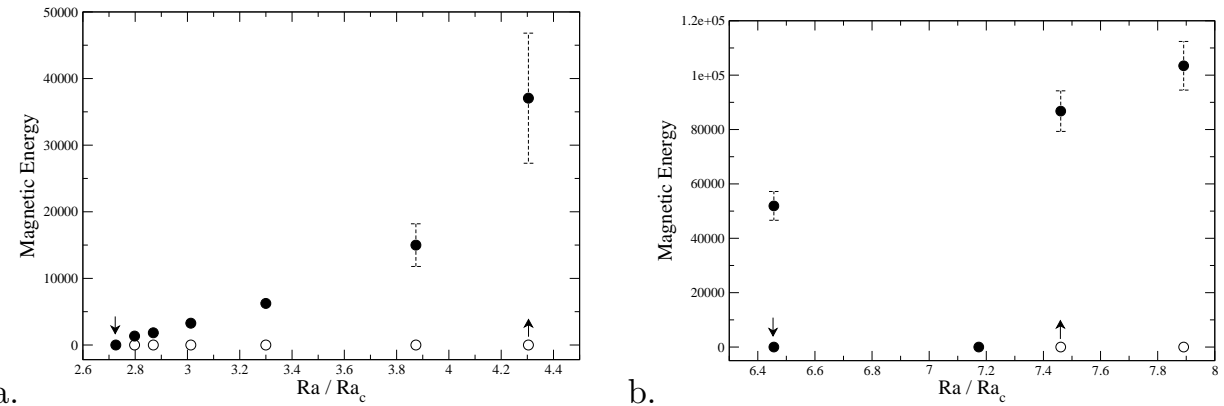

Fig. 6. Mean magnetic energy versus Rayleigh number. These supercritical (a) and subcritical (b) bifurcations were obtained respectively for $\mathrm{E}=10^{-4}$ and $\mathrm{Pm}=3$ and $\mathrm{E}=10^{-4}$ and $\mathrm{Pm}=0.67$. Error bars indicate the standard deviation of the magnetic energy fluctuations around its mean value.

By increasing the Ekman number at fixed magnetic Prandtl number, we therefore successively described supercritical, subcritical and isola bifurcation diagrams. We further extended our study to $\mathrm{E}=10^{-4}$ and $\mathrm{Pm}=0.67$ (figure [6., b) and obtained a subcritical bifurcation. 


\subsection{Interpretation}

We have studied different bifurcations obtained for Ekman number values ranging from $10^{-3}$ to $10^{-4}$, magnetic Prandtl number values from 0.67 to 6 and Rayleigh number values from $\mathrm{Ra} \simeq 2 \times \mathrm{Ra}_{c}$ to $\mathrm{Ra} \simeq 10 \times \mathrm{Ra}_{c}$. In this parameter regime, the evolution of the bifurcation diagram is summarised in figure 7 For a given Ekman number, a supercritical bifurcation (top plot) is obtained for a sufficiently high value of the magnetic Prandtl number. By decreasing its value, it is possible to obtain a subcritical bifurcation (middle plot), which may exhibit unusual features, such as re-stabilization of the purely hydrodynamical state. An unstable branch therefore must exist, it could be connected, for larger values of the Rayleigh number, to the stable branch corresponding to dynamo solutions. If the magnetic Prandtl number is further decreased, the range of Rayleigh number for which the non dynamo solution is unstable vanishes. An isola is then obtained (bottom plot), in this situation the purely hydrodynamical solution is always stable. The very same sequence is obtained by increasing $\mathrm{E}$ at fixed $\mathrm{Pm}$, as the dipolar domain (domain II on figure 1) shifts towards lower values of $\mathrm{Pm}$ as $\mathrm{E}$ is decreased.

\subsection{Feedback and coupling}

Let us now turn to the effects of the saturated magnetic field on the flow. We compare on figure 8 simulations obtained for $\mathrm{E}=1 \cdot 10^{-4}$ and $\mathrm{Pm}=3$, before and after saturation. The magnetic field clearly acts here to inhibit thermal convection. Not only does it lower the averaged value of the kinetic energy, but it also reduces the amplitude of fluctuations. This behavior is typical of the parameter space we investigated, there are however a few noticeable exceptions.

In the case of the subcritical bifurcation obtained for $\mathrm{E}=3 \cdot 10^{-4}$ and $\mathrm{Pm}=3$, we can investigate a range of Rayleigh numbers from $\mathrm{Ra}=3.16$ to $3.30 \times \mathrm{Ra}_{c}$ (which are lower than the linear threshold value). It is then found, figure9, that the presence of the magnetic field yields stronger velocity fluctuations. Indeed the purely hydrodynamical solution (without magnetic field) is presented on the right of each graph (either after a loss of dynamo action in the metastable case $\mathrm{Ra}=3.16 \times \mathrm{Ra}_{c}$, or through an independent non-magnetic simulation).

Dynamo solutions corresponding to the isola branch described for $\mathrm{E}=10^{-3}$ and $\mathrm{Pm}=3$ are metastable. Magnetic energy fluctuations are here comparable to the mean value, which leads to a loss of dynamo action after a time which depends on the Rayleigh number as well as on the particular choice of initial conditions. We should highlight a simulation performed with a Rayleigh number $\mathrm{Ra}=3.76 \times \mathrm{Ra}_{c}$, presented on figure 10. Dynamo action was here maintained for $140 \tau_{\nu}$ (with $\tau_{\nu}=L^{2} / \nu$ ) before being lost, this corresponds to $20 \tau_{\eta}$ (with $\tau_{\eta}=r_{o}^{2} / \eta$ ), and to $\simeq 200$ dipole decay time $\left(\tau_{d}=\tau_{\eta} / \pi^{2}\right)$. Such behaviour raises questions as to "how long should a dynamo be investigated to assess its stability?". Once lost, the dynamo solution cannot be recovered, as the purely hydrodynamic solution is stable for this parameter regime. Once in the basin of attraction of the $\mathbf{B}=\mathbf{0}$ state, the field cannot recover. This is 

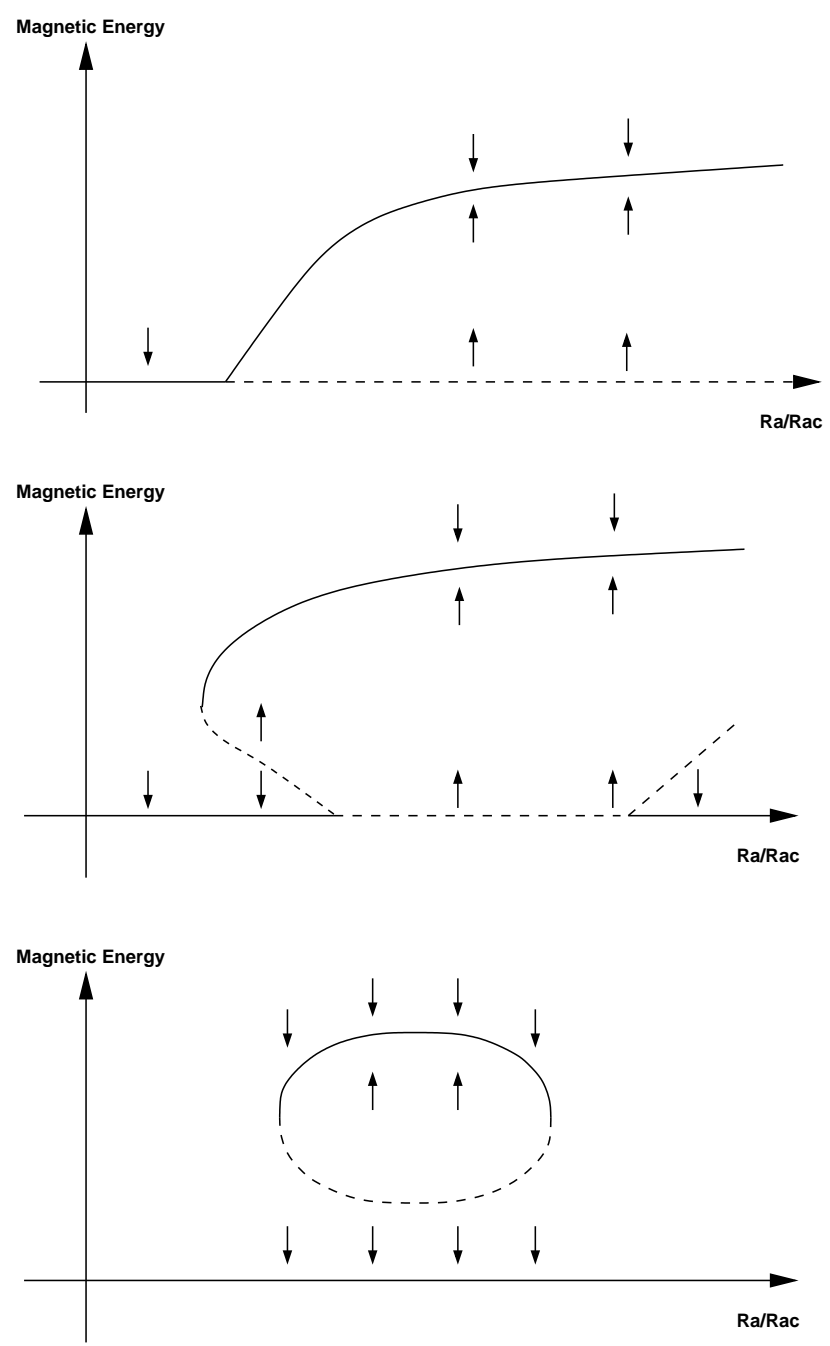

Fig. 7. Summary of the typical bifurcations obtained numerically. As the magnetic Prandtl number is decreased at fixed Ekman number one obtains successively supercritical (top), subcritical (middle) with restabilisation, and isola (bottom). The same sequence is achieved by increasing the Ekman number at fixed Rossby number. The dashed lines indicating unstable branches are here speculative, except for the $\mathbf{B}=\mathbf{0}$ state.

due to the multiplicative character of hydrodynamic fluctuations in the induction equation. The effect of the hydrodynamic fluctuations on the field vanishes near $\mathbf{B}=\mathbf{0}$.

Let us now turn to the statistical properties of the fluctuations of kinetic and magnetic energies. Figure 12 reports the probability density functions (PDF) of both energies for $\mathrm{Pm}=3$ and on the top row $\mathrm{E}=3.10^{-4}, \mathrm{Ra}=3.7 \times \mathrm{Ra}_{c}$ (subcritical bifurcation), on the bottom row $\mathrm{E}=10^{-4}, \mathrm{Ra}=3.3 \times \mathrm{Ra}_{c}$ (supercritical 

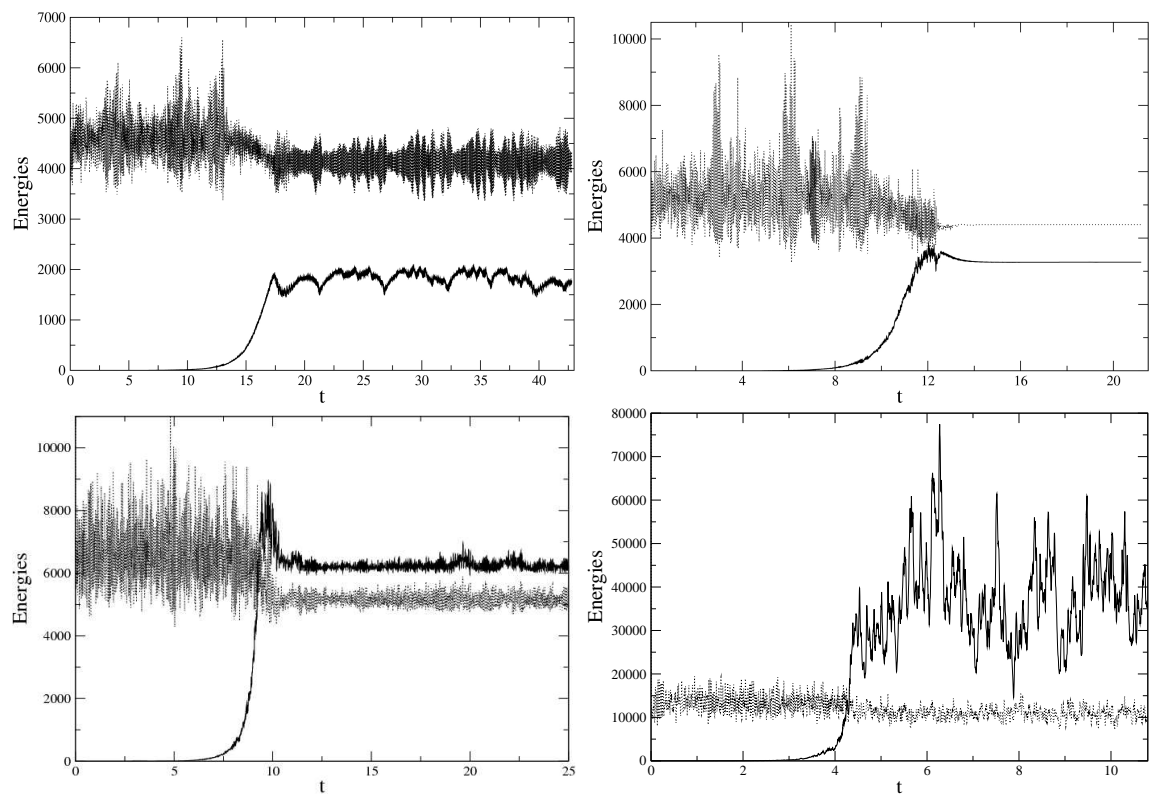

Fig. 8. Magnetic energy (solid lines) and kinetic (dashed lines) as a function of time for $\mathrm{E}=$ $1.10^{-4}, \mathrm{Pm}=3$ and from left to right and top to bottom $\mathrm{Ra}=2.87,3.01,3.30$ and $4.30 \times \mathrm{Ra}_{c}$.

bifurcation). In both cases the PDFs of the magnetic energy are much less symmetrical than their counterpart on the kinetic energy. This is a robust characteristic, which was observed in all our simulations.

This appears again to be a direct consequence of the multiplicative character of the hydrodynamic fluctuations in the induction equation. Whereas temperature fluctuations act as additive noise on the velocity equation, velocity fluctuations act as a multiplicative noise on the magnetic field (i.e. a term proportional to $\mathbf{B}$ ). One can show on a very simple system how a multiplicative white noise (with symmetric PDF) yields a chi-2 law (asymmetric PDF) for the variable affected by the noise. Let us consider the temporal evolution of a scalar, for a conservative system, we can write

$$
\frac{\mathrm{d} X}{\mathrm{~d} t}=-\frac{\mathrm{d} G}{\mathrm{~d} X} .
$$

An additive noise would drive fluctuations of $X$ within a fixed potential $G$, a multiplicative noise will instead modify the shape of the potential. Let us consider, for example, the case of a steady subcritical bifurcation in the presence of a multiplicative noise. The normal form (assuming the $X \mapsto-X$ invariance) yields a potential of the form

$$
G(X)=-\frac{\mu+\xi(t)}{2} X^{2}-\frac{\alpha}{4} X^{4}+\frac{1}{6} X^{6} .
$$

In the absence of noise $(\xi \equiv 0)$, solutions to (445) are: the trivial $X=0$ (stable for $\mu<0$ unstable otherwise), and the four roots of $\mu+\alpha X^{2}-X^{4}$, for $-\alpha^{2} / 4 \leqslant \mu<0$. 

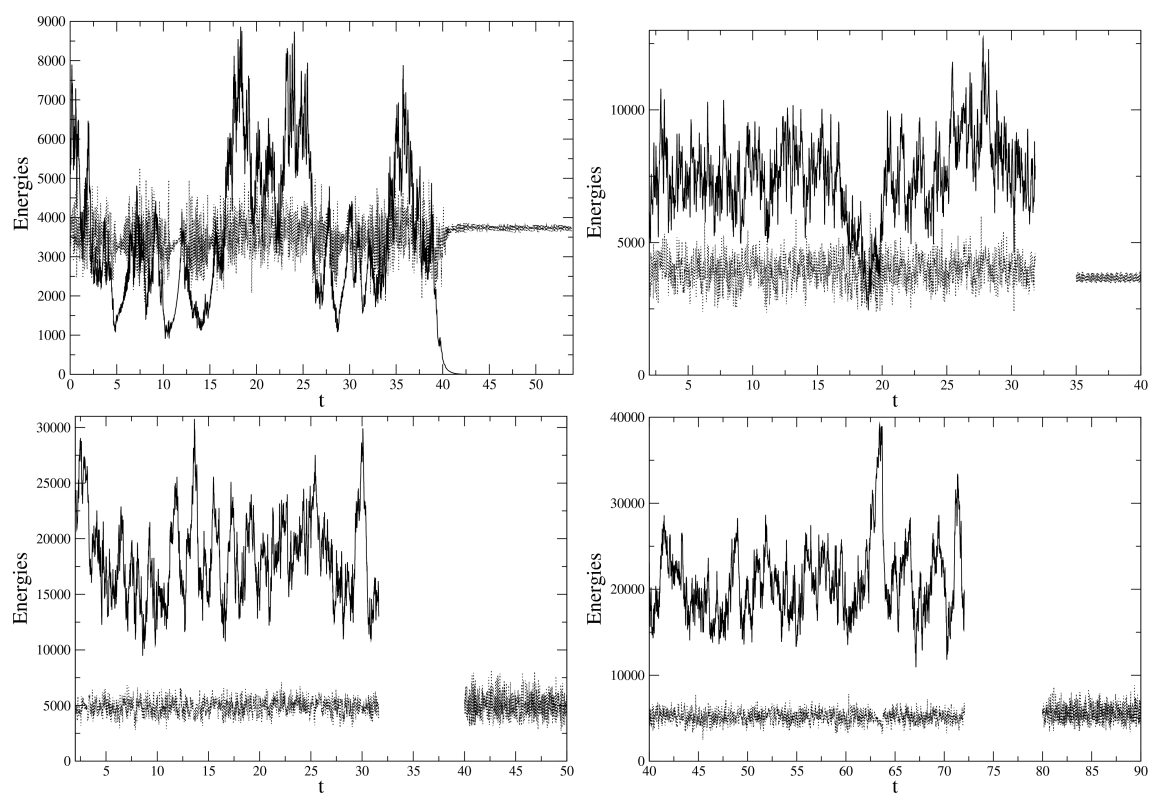

Fig. 9. Magnetic (solid) and kinetic (dashed) energies as a function of time for $\mathrm{E}=3 \cdot 10^{-4}$ and $\mathrm{Pm}=3$ and from top to bottom and left to right $\mathrm{Ra}=3.16,3.30,3.70$ et $3.85 \times \mathrm{Ra}_{c}$. For all graphs, the signal on the far right part of the graph corresponds to the kinetic energy evolution in the absence of magnetic field (purely hydrodynamic solution).
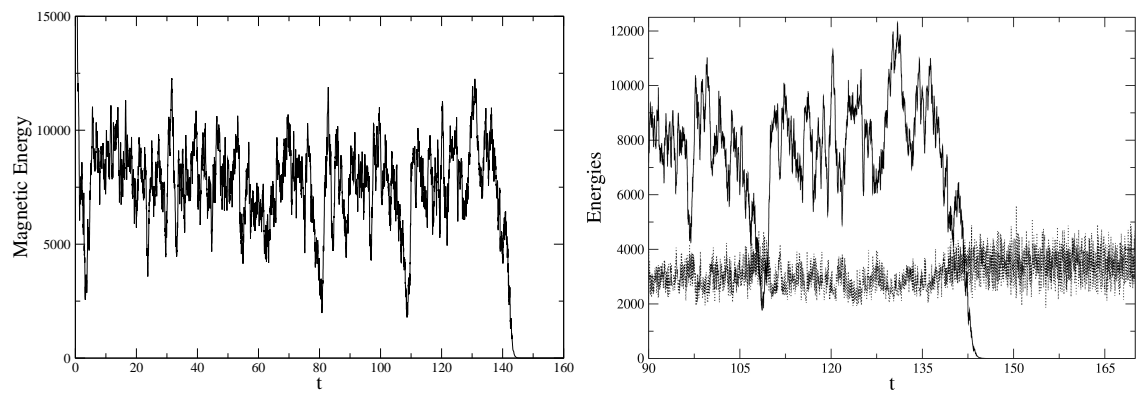

Fig. 10. Magnetic (and kinetic on the right graph in dashed lines) energies versus time for $\mathrm{E}=$ $10^{-3}, \mathrm{Pm}=3$ and $\mathrm{Ra}=210$. The dynamo solution is lost after some $140 \tau_{\nu}$. Both the mean and the fluctuating kinetic energies increase after the magnetic field collapse.

Two of these are unstable, the two others being stable (subcritical branch) and continuous for $\mu \geqslant 0$.

Let us now consider the subcritical branch of this system with noise. Below the threshold, the parameter $\mu$ is negative. Figure 11 examplifies the shape of the potential $G(X)$ for different values of $\xi(t)$, and thus of the prefactor of the $X^{2}$ term. When it is low enough (solid line), there exists a negative minimum. This minimum 
provides the preferred state of the system. When $\xi(t)$ becomes large enough, the potential can fluctuate until both minima of the potential reach the same value (dotted curve). If the prefactor of the $X^{2}$ term becomes even larger, the potential minimum associated to non-zero $X$ becomes local and the prefered state of the system becomes $X=0$.

It is important to appreciate that $X=0$ is an absorbing state. Indeed the noise being multiplicative will not affect the system any longer when $X=0$. If fluctuations are large enough, non-trivial solutions of a subcritical system in the presence of multiplicative noise can thus be destabilised through a "large" fluctuation. The trivial solution then becomes permanent. This simpler model explains the behaviour reported above in our dynamo simulations.

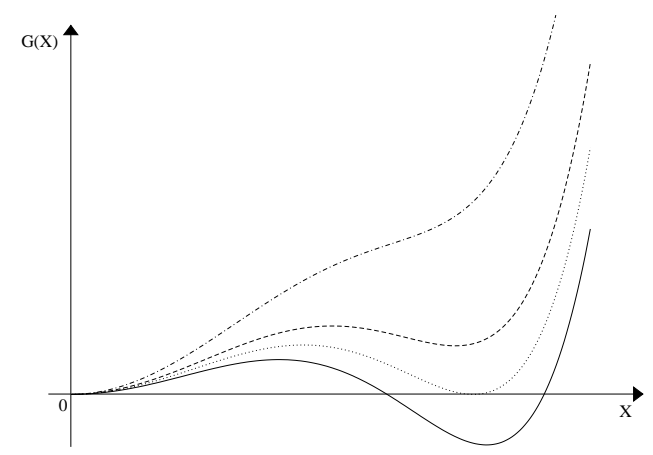

Fig. 11. Potential $G(X)$ as a function of the order parameter $X$. The potential represented here takes the form (5) setting $\alpha=5$ and the prefactor of the $X^{2}$ term to 2 (solid line), 2.3435 (dotted), 2.7 (dashed) et 3.7 (dot-dashed).

The presence of a multiplicative noise implies that previously well defined equilibrium values (in the absence of noise) become probabilities. Let us consider a simple supercritical example of normal form

$$
\frac{\mathrm{d} X}{\mathrm{~d} t}=[\mu+\xi(t)] X-X^{3} .
$$

If one assumes that $\xi(t)$ is a white and gaussian noise of variance $D$, one can then show that the equation governing the evolution of the probability $P$ of $X$ to have a given value is

$$
\frac{\partial P}{\partial t}=-\frac{\partial}{\partial X}\left[\left(\mu X-X^{3}\right) P\right]+\frac{D}{2} \frac{\partial}{\partial X}\left(X \frac{\partial}{\partial X} X P\right) .
$$

Integrating this equation in $X$ for steady probabilities, yields

$$
J=\left(-\mu X+X^{3}\right) P+\frac{D}{2}\left(X \frac{\partial}{\partial X} X P\right) .
$$


The right-hand-side vanishes for $X=0$, which implies that $J=0$. The change of variable $q=X P$ provides

$$
\frac{\partial q}{\partial X}=\frac{2}{D}\left(\frac{\mu}{X}-X\right) q, \text { and thus } P=C|X|^{2(\mu / D)-1} e^{-X^{2} / D} .
$$

It is worth noting that starting from a multiplicative gaussian white noise (thus with a symmetric probability distribution), one obtains a non-symmetric probability distribution for the variable $X$.

One can show in a similar manner that for a subcritical bifurcation with a normal form given by (445), the probability $P(X)$ to meet a given value is

$$
P=C|X|^{2(\mu / D)-1} e^{X^{2} / D-X^{4} / 2 D} .
$$

This simple example can, off course, not account for the full complexity of the coupled non-linear MHD system, yet it demonstrates how the structure of the induction equation accounts for the tendency for a stronger asymmetry in PDFs of the magnetic than in the kinetic energy (see figure 12).

a.
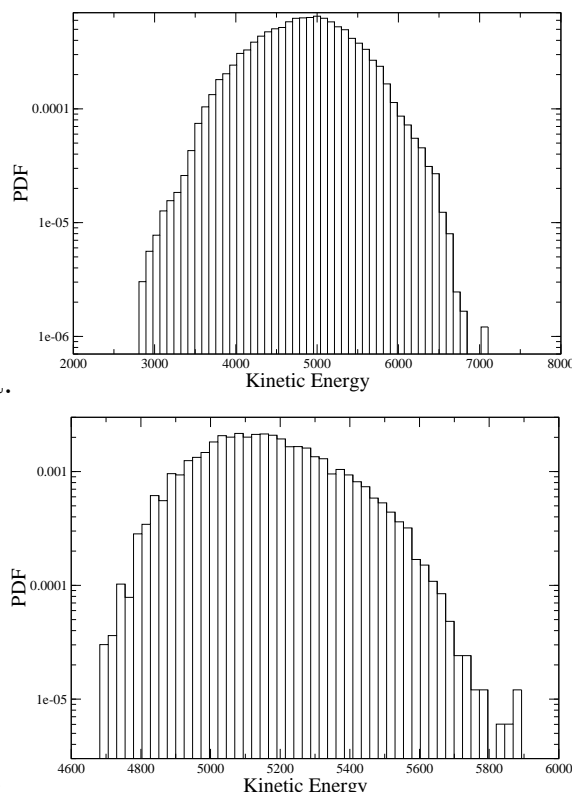

b.

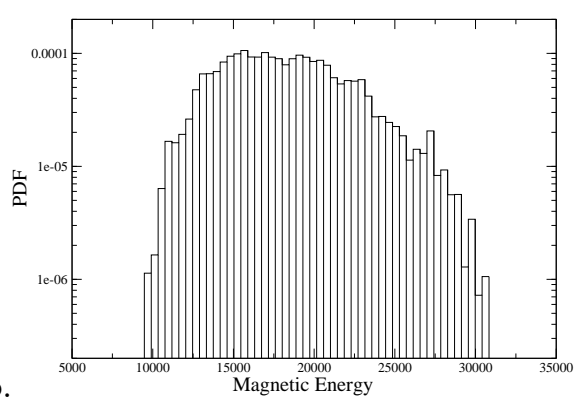

d.

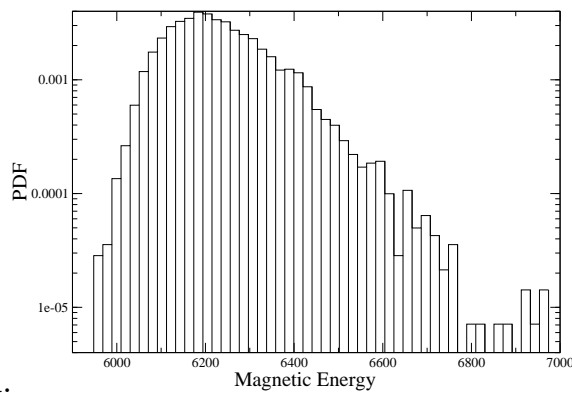

Fig. 12. Probability Density Functions for the kinetic energy (left) and magnetic energy (right), for $\mathrm{Pm}=3$ and $\mathrm{E}=3.10^{-4}, \mathrm{Ra}=3.7 \times \mathrm{Ra}_{c}$ (top), $\mathrm{E}=10^{-4}, \mathrm{Ra}=3.3 \times \mathrm{Ra}_{c}$ (bottom). The top line corresponds to a subcritical bifurcation, the bottom one to a supercritical bifurcation.

\subsection{Multiple Solutions}

In the course of the investigation reported here, we did not encounter proper multiple co-existing branches of dynamo action (but see section 3 ). We however observed 
an interesting behaviour which happens to be directly related to the non-uniqueness of the purely hydrodynamic solution itself. Both the magnetic and the kinetic energies are presented for $\mathrm{E}=3.10^{-4}, \mathrm{Pm}=6$ and $\mathrm{Ra}=2.57 \times \mathrm{Ra}_{c}$ in figure 13, Two different simulations, which only differ by a minute initial fluctuation, are presented on this figure. The behaviour is rather unexpected and untypical. While the simulations are first similar, the dynamo solution appears to reach a stable state (damped oscillations) but then violently destabilises. In one case dynamo action is maintained (left), but it is lost in the other one (right).
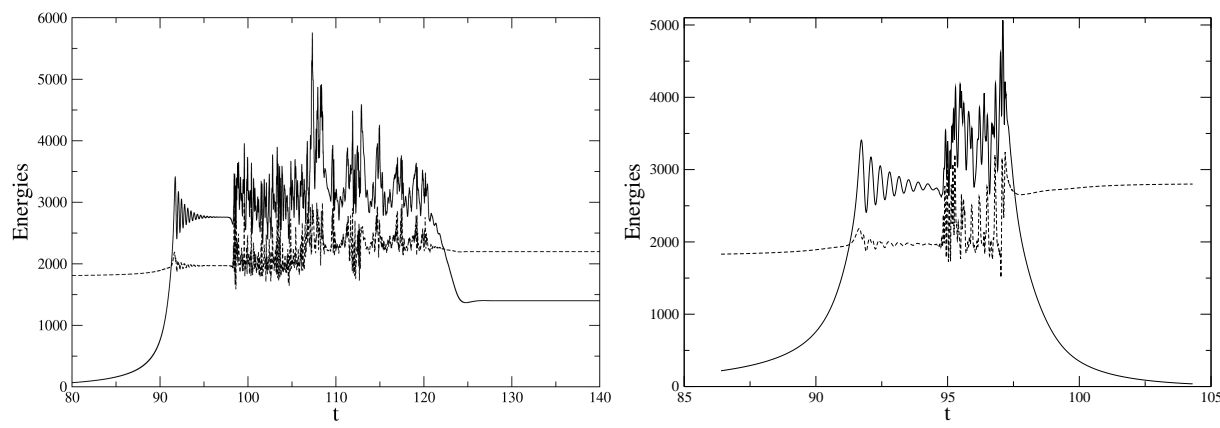

Fig. 13. Magnetic (solid) and kinetic (dashed) energies versus time for $\mathrm{E}=3.10^{-4}, \mathrm{Pm}=6$ and $\mathrm{Ra}=2.57 \times \mathrm{Ra}_{c}$. Left and right graphs correspond to two independent simulations with initial conditions which differ only from a very small perturbation (identified in the text as "run 1" and "run 2").

For this parameter space, we identified at least two stable solutions to the purely hydrodynamic problem. Further more, we observed (figure 14), a modification in time of the dominant azimuthal Fourier mode both for the kinetic and magnetic fields. Simulations were initialised with a random perturbation of the magnetic and temperature fields and a fluid at rest. The chosen parameters are here extremely close to the threshold value, and the magnetic field growth is slow (the growthrate is 0.058 ). Thermal convection therefore had enough time to develop largely uninfluenced by the Lorentz force. For this parameter regime the kinetic energy of the most unstable mode of hydrodynamic convection is steady in time and the dominant modes are $m=6$ and $m=0$. When the field reaches saturation is has the same structure as the flow, dominated by an $m=6$ symmetry. This mode however quickly destabilises and, as illustrated on figure 14 after a competition between the $m=4,5$ and 6 modes, the dominant modes becomes (both for the kinetic and the magnetic energy) $m=5$ for the first run and $m=4$ for the second one. The simulation "run 2", corresponds to a loss of dynamo action, as the $m=4$ convection mode does not appear able to sustain the field against diffusion. Both simulations ("run1" and "run 2") were carried further in time for another $30 \tau_{\nu}$, to assess the stability of their final state.

This intricate behaviour therefore comes as the consequence of the existence of 

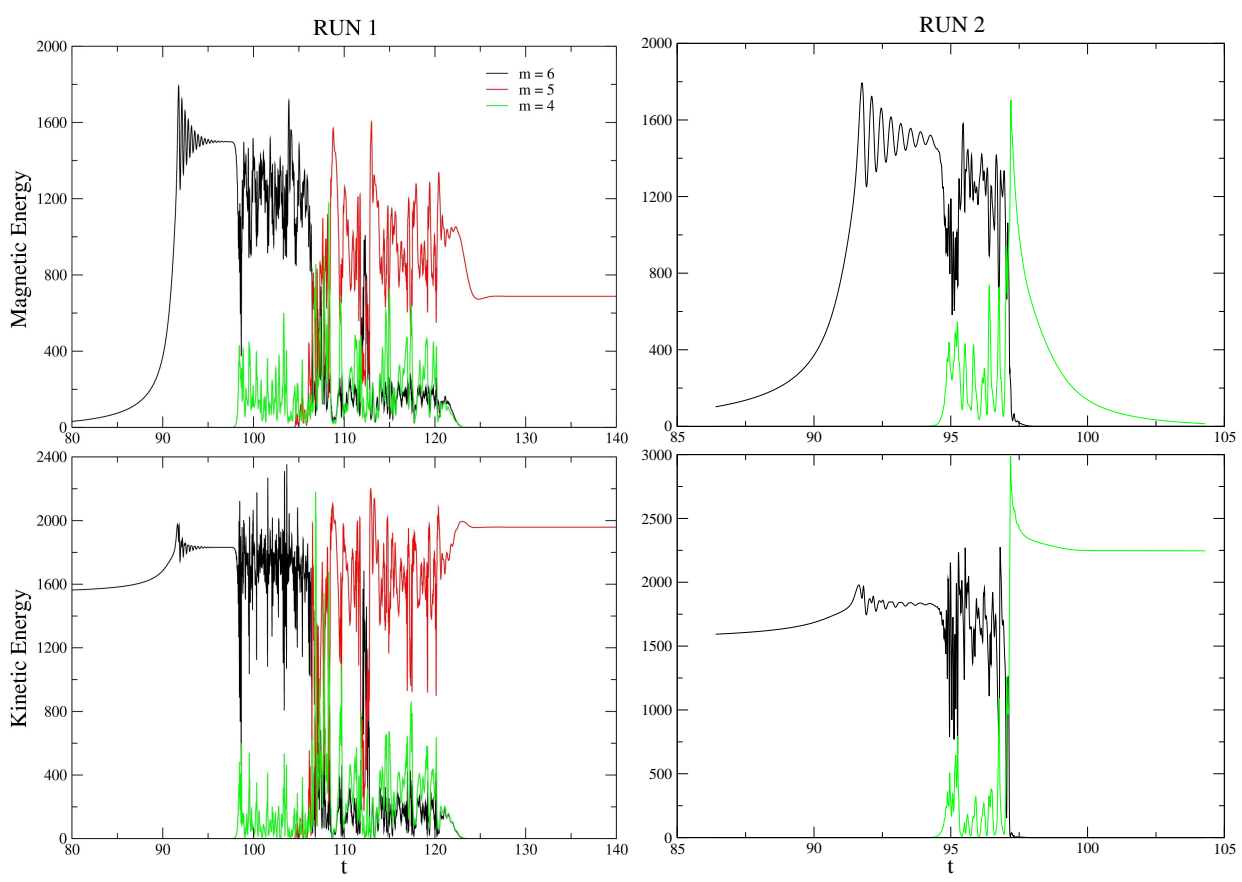

Fig. 14. Magnetic (top) and kinetic (bottom) energies corresponding to the azimuthal Fourier modes $m=4,5$ and 6 for the simulations presented on figure 13

three possible modes of hydrodynamic convection (all stables). The fastest growing mode, $m=6$ does act as a kinematic dynamo, but this dynamo is non-linearly unstable and destabilises either to the $m=4$ or to the $m=5$-mode. The later is also a dynamo, but contrary to $m=6$ it is a stable solution. The $m=4$-mode does not act as dynamo, the magnetic field is then lost. We did not observe any heteroclynic cycle. The behavior reported here is reminiscent of self-killing dynamos as described in [10].

\section{Further developments}

We presented here a study of the onset of dynamo action over a wide parameter range. We should note however that one parameter was, for simplicity, kept constant throughout the entire study: the Prandtl number $(\operatorname{Pr}=\nu / \kappa)$. It would be interesting to know how this parameter affects the picture presented here.

Since this work was performed and published in french in $2005[\underline{6}$, we have further investigated the parameter space towards large values of the magnetic Prandtl number. Yet another kind of bifurcation diagram has been produced. It is characterized by two co-existing branches of different energies. These results will be presented and discussed elsewhere [11. 
Morin \& Dormy

\section{Acknowledgements}

The authors are very grateful to Stephan Fauve for many fruitful discussions in the course of this work.

\section{References}

1. C. Kutzner \& U. Christensen, From stable dipolar towards reversing numerical dynamos, Phys. Earth Planet. Int., 131, 29, 2002.

2. U. Christensen \& J. Aubert, Scaling properties of convection-driven dynamos in rotating spherical shells and application to planetary magnetic fields, Geophys. J. Int. 166, 97-114, 2006.

3. C. Kutzner, Untersuchung von Feldumkehrungen an einem numerischen Modell des Geodynamos, PhD thesis, Göttingen University, 2003.

4. U. Christensen, P. Olson \& G. Glatzmaier, Numerical modeling of the geodynamo: a systematic parameter study, Geophys. J. Int. 138, 393, 1999.

5. E. Dormy \& J.-L. Le Mouël, Geomagnetism and the dynamo: where do we stand?, C.R.Physique, 9, 711-720, 2008.

6. V. Morin, Instabilités et bifurcations associées à la modélisation de la Géodynamo, $\mathrm{PhD}$ thesis, Université Paris VII (2005), (http://tel.archives-ouvertes.fr/tel-00011484/en/).

7. Morin V., Dormy E., "Numerical investigation of the dynamo bifurcation", FG.00008, APS, Division of Fluid Dynamics, 59th annual meeting, Tampa, USA, Nov. 2006; Morin V., Dormy E., "Dynamo bifurcation in geodynamo models", \#DI31A-0255, AGU Fall Meeting, San Francisco, USA, Dec 2007; Dormy E., workshop "Modelling geophysical systems by statistical mechanics methods", Erice, Italy, May 2008; Morin V., Dormy E., "Numerical investigation of the dynamo bifurcation in geodynamo models", EURO MHD, Nice, France, sept. 2008; Dormy E., workshop "Waves and instabilities in geophysical and astrophysical flows", Porquerolles, France, May 2009.

8. Y. Ponty, J.P. Laval, B. Dubrulle, F. Daviaud, J.F. Pinton, PRL, 99, 224501, 2007; R.D. Simitev, F.H. Busse, EPL, 85, 19001, 2009.

9. Christensen et al, Phys. Plan. Earth Int. 128, 25-34, 2001.

10. H. Fuchs, K.H. Rädler, M. Reinhardt, in Dynamo and Dynamics, Chossat, Armbuster \& Oprea Eds, Kluwer 2001.

11. V. Morin \& E. Dormy, Weak and Stong Field Dynamos, 2009 submitted. 